\title{
Re-Envisaging the Eight Developmental Stages of Erik Erikson: The Fibonacci Life-Chart Method (FLCM)
}

\author{
Robert G. Sacco ${ }^{1}$ \\ ${ }^{1}$ School of Behavioral and Health Sciences, Northcentral University, Arizona, USA \\ Correspondence: Robert G. Sacco, School of Behavioral and Health Sciences, Northcentral University, Prescott \\ Valley, AZ, 86314, USA. E-mail: robgsacco@gmail.com
}

Received: February 9, 2013

Accepted: March 19, 2013 Online Published: March 29, 2013

doi:10.5539/jedp.v3n1p140

URL: http://dx.doi.org/10.5539/jedp.v3n1p140

\begin{abstract}
The purpose of this study is to describe the use of Fibonacci numbers to model Erikson's eight developmental stages and to formulate practical clinical implications. Using a new method, called the Fibonacci Life-Chart Method (FLCM), all prospective dates based on the Fibonacci sequence between January 1, 2000 and December 31, 2100 were identified. This study found the FLCM produced a developmental pattern characterized by eight recognizable stages. This finding constitutes a new classification of Erikson's eight developmental stages. The present research provides support for Erikson's epigenetic view of predetermined, sequential stages to human development based on the occurrence of Fibonacci numbers in biological cell division and self-organizing systems. This method may help identify populations at risk for psychological disorder, which would allow early intervention. However, a longitudinal study is required to establish its predictive power.
\end{abstract}

Keywords: developmental stages, dynamic systems theory, erikson, fibonacci numbers

\section{Introduction}

Overall, Erikson's greatest contribution to research on human development is his life-cycle theory and its eight stages. Erikson (1982) maintained that within the span of a lifetime, individuals advance through a series of eight developmental stages, each characterized by a unique psychological issue. The degree of resolution (or unresolution) of each stage forms the characteristics of individual personality and impacts the degree of resolution (or unresolution) of later stages. Erikson defined the following eight developmental stages: trust vs. mistrust, autonomy vs. shame and doubt, initiative vs. guilt, industry vs. inferiority, identity vs. identity confusion, intimacy vs. isolation, generativity vs. stagnation, and ego integrity vs. despair, which are related to the following ages: early infancy $\left(\begin{array}{lll}1 & 1 / 2\end{array}\right)$, toddler $\left(\begin{array}{ll}1 & 1 / 2\end{array}-3\right)$, early childhood (3-6), middle childhood (6-12), adolescence (12-18), young adulthood (19-40), middle adulthood (40-65), and older adulthood (65+). These stages and their associated personality outcomes are summarized in Table 1.

Table 1. Erikson's eight stages of psychosocial development

\begin{tabular}{llll}
\hline Stage & Period & Personality Attributes & Age \\
\hline 1 & Early Infancy & Trust vs. Mistrust & $1-1 \frac{1 / 2}{2}$ \\
2 & Toddler & Autonomy vs. Shame and Doubt & $1 \frac{1}{2}-3$ \\
3 & Early Childhood & Initiative vs. Guilt & $3-6$ \\
4 & Middle Childhood & Industry vs. Inferiority & $6-12$ \\
5 & Adolescence & Identity vs. Identity Confusion & $12-18$ \\
6 & Young Adulthood & Intimacy vs. Isolation & $19-40$ \\
7 & Middle Adulthood & Generativity vs. Stagnation & $40-65$ \\
8 & Older Adulthood & Integrity vs. Despair & $65+$ \\
\hline
\end{tabular}

Note. This information is from Erikson (1982). 
Each of these stages has a biological foundation in an individual's physical maturation and cognitive development. Erikson used the term "epigenesis" to describe the organic quality of this developmental model. Assimilated from embryology, the word describes how fetal organs normally develop in a careful sequential priority with one another. Similarly, each of Erikson's psychosocial stages builds on the other, as a resolution to a particular psychosocial crisis, and is, consequently, positively balanced. The human body, including skin, eyes, limbs, internal organs, and central nervous system rest on genetic and proteomic codes. However, genetics depends on essentially physico-chemical processes, and so must meet further basic non-genetic constraints. Physical and chemical constraints are the parameters of a physical and biological universe establishing an inherent epigenetic stage of formativeness on which numerous forms have possibilities for emergence. The problem is that Erikson's life-cycle approach, derived from the biological principle of epigenesis, has not considered physico-chemical parameters.

\subsection{A Theoretical Framework: Human Developmental Stages and the Fibonacci Sequence}

A priori optimum development in organisms, from single cell to multicellular organisms, or from skeletal cells to a skeleton or limbs exemplify Fibonacci patterns. These developmental patterns consist of two primary characteristics: (1) a number of the organisms structural arrangements may fall into the series $1,1,2,3,5,8$, . or (2) logarithmic growth based on the ratio of consecutive Fibonacci numbers $(1.618033988 \ldots$, also known as the golden ratio or $\varphi$ ). The growth patterns observed occur throughout nature in the arrangement of skin pores in tetrapods, the spiral shape of snails and sea shells, and the overall structure of plants. Fibonacci numbers occur in atoms and electrons (Huntley, 1969), the DNA molecule (Wahl, 1988), biological cell division (Spears \& Bicknell-Johnson, 1998), models of growth and death (Hoggatt \& Lind, 1969), bronchial airway segment bifurcations (Goldenberger, West, Dresselhaus, \& Bhargava, 1985), experimental growth of tumor nodules (Prokopchuk, 1981), and many other aspects of human biology (e.g., position of facial features, body proportions).

Waskom (1972) pointed out that human developmental stages might follow the Fibonacci sequence. To describe human development with the Fibonacci sequence, Waskom simply imagined Fibonacci numbers as representing age markers (in years) for cycles or stages of development. Thus it was asserted the numbers that mark human developmental stages are the same numbers expressed in the Fibonacci sequence. This became the foundation of Waskom's model of human development. Rose (1991) expanded on it somewhat for uniformity of demonstration through the life cycle. Under this scheme, Waskom delineated eight stages of the life cycle associated with the following ages: early infancy $(0-1)$, toddler (1-5), early childhood (5-8), middle childhood (8-13), adolescence (13-21), young adulthood (21-34), middle adulthood (34-55), and older adulthood (55+).

One criticism of Waskom's classification is whether Fibonacci numbers must be regarded as numeric variables in years. This rule gives each number in the Fibonacci sequence an average multiple of 365 days. The purpose of the present study is to identify a more analytical classification of the Fibonacci sequence in relation to Erikson's eight developmental stages. It was hypothesized the Fibonacci sequence based on a multiple of the 24-hour day/night cycle could help clarify Erikson's eight developmental stages. This hypothesis was examined by a new methodology called the Fibonacci Life-Chart Method (FLCM).

\section{Methodology}

Previous models of human development have regarded Fibonacci numbers as numeric variables in years (Rose, 1991; Waskom, 1972). However, a more precise numeric designation for each number in the Fibonacci sequence is perhaps the 24-hour day. First, biological cycles are defined by daily changes synchronized or entrained to the 24-hour rotation of the earth (Roenneberg \& Foster, 1997). To keep these rhythms in proper alignment with the day/night cycle, all living organisms have adapted by evolving their internal clockwork tuned to a 24-hour day/night cycle to adapt their behavior, physiology, and metabolism.

Second, the digital roots of the Fibonacci sequence produce an infinite series of 24 repeating numbers (Meisner, 2012). Further, the 24-repeating pattern follows an approximate sinusoidal pattern (Figure 1). While the discrete-time system numbers 12,30, 60, and 360 are all based on the dodecahedron and golden ratio (Stakhov, 2009), only the day relates to the 24-repeating sinusoidal pattern, since one day is equal to 24 ( $2 \times 12)$ hours. 


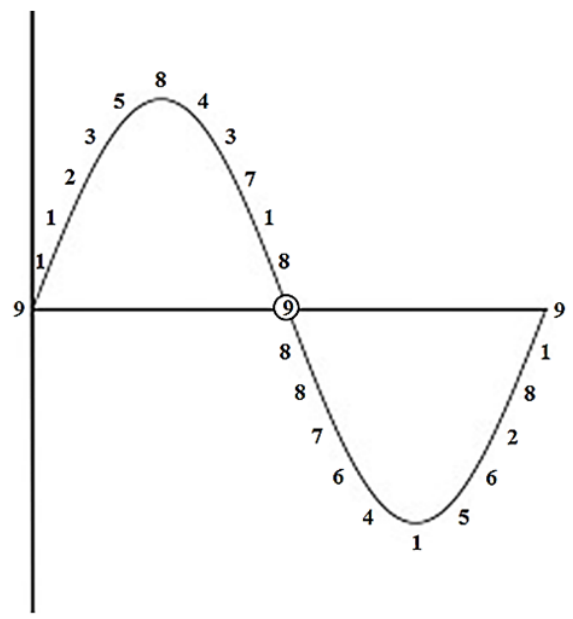

Figure 1. Digital roots of the 24-repeating pattern

Note. The 24 repeating digital roots of the Fibonacci sequence are: 1, 1, 2, 3, 5, 8, 4, 3, 7, 1, 8, 9, 8, 8, 7, 6, 4, 1, 5, 6, 2, 8, 1, 9 (Meisner, 2012). Numbers 7 and 8 and 2 and 1 are the only numbers not fitting the sinusoidal pattern.

Thus, to examine the relationship between the Fibonacci sequence and Erikson's eight developmental stages, a new method, called the Fibonacci Life-Chart Method (FLCM) was developed. This method is a type of growth modeling that is used to identify developmental patterns by representing Fibonacci numbers as numeric multiples of 24-hours.

\subsection{The Fibonacci Life-Chart Method}

The Fibonacci Life-Chart Method (FLCM) is a method for identifying developmental patterns comprising the steps of: (a) selecting a birthdate which to apply the Fibonacci sequence; (b) calculating primary Fibonacci-based time projections wherein the Fibonacci-sequence is added to the birthdate with Fibonacci numbers representing days; and (c) calculating secondary Fibonacci-based retrospective and prospective dates wherein the derived Fibonacci dates from step two are multiplied by the Fibonacci constants or ratios 0.618, 1.618, 0.786 (square root of 0.618 ), and 1.27 (square root of 1.618). For the present study, the model has been kept as simple as possible by not including step three in the analysis of future time projections. Step three does not alter the predictive utility of the primary prospective dates in the model.

\section{Results}

Table 2 shows the results of the FLCM. As can be seen, the FLCM produces a developmental pattern characterized by eight recognizable stages. This finding constitutes a new classification of Erikson's eight developmental stages: early infancy (1-2), toddler (2-4), early childhood (4-7), middle childhood (7-11), adolescence (11-18), young adulthood (18-29), middle adulthood (29-48), and older adulthood (48-78+) (Table $3)$. The eight stages closely match the well-known Lucas series $(1,3,4,7,11,18,29,47,76$, etc.). The Lucas series has the same characteristic of Fibonacci numbers whereby each integer is the sum of the two previous integers. The Lucas series of numbers is found in the number of cells in each cycle of cell division (Jovanovic, 2003).

Table 2. Fibonacci Life-Chart Method

\begin{tabular}{lll}
\hline Fibonacci Numbers & Date & Age \\
\hline 0 & $1 / 1 / 2000$ & 0 \\
1 & $1 / 2 / 2000$ & 0 \\
1 & $1 / 3 / 2000$ & 0 \\
2 & $1 / 5 / 2000$ & 0 \\
3 & $1 / 8 / 2000$ & 0 \\
\hline
\end{tabular}




\begin{tabular}{lll}
\hline 5 & $1 / 13 / 2000$ & 0 \\
8 & $1 / 21 / 2000$ & 0 \\
13 & $2 / 3 / 2000$ & 0 \\
21 & $2 / 24 / 2000$ & 0 \\
34 & $3 / 29 / 2000$ & 0 \\
55 & $5 / 23 / 2000$ & 0 \\
89 & $8 / 20 / 2000$ & 0 \\
144 & $1 / 11 / 2001$ & 1 \\
233 & $9 / 1 / 2001$ & 1 \\
377 & $9 / 13 / 2002$ & 2 \\
610 & $5 / 15 / 2004$ & 4 \\
987 & $1 / 27 / 2007$ & 7 \\
1597 & $6 / 12 / 2011$ & 11 \\
2584 & $7 / 9 / 2018$ & 18 \\
4181 & $12 / 19 / 2029$ & 29 \\
6765 & $6 / 27 / 2048$ & 48 \\
10946 & $6 / 16 / 2078$ & 78 \\
\hline
\end{tabular}

Note. Fibonacci Numbers represent 24-hour days.

Table 3. Erikson's re-envisaged eight stages of psychosocial development

\begin{tabular}{llll}
\hline Stage & Period & Personality Attributes & Age \\
\hline 1 & Early Infancy & Trust vs. Mistrust & $1-2$ \\
2 & Toddler & Autonomy vs. Shame and Doubt & $2-4$ \\
3 & Early Childhood & Initiative vs. Guilt & $4-7$ \\
4 & Middle Childhood & Industry vs. Inferiority & $7-11$ \\
5 & Adolescence & Identity vs. Identity Confusion & $11-18$ \\
6 & Young Adulthood & Intimacy vs. Isolation & $18-29$ \\
7 & Middle Adulthood & Generativity vs. Stagnation & $29-48$ \\
8 & Older Adulthood & Integrity vs. Despair & $48-78+$ \\
\hline
\end{tabular}

\section{Discussion}

The present study investigated the relation between the Fibonacci Life-Chart Method (FLCM) and Erikson's eight stages of development. The results of this study provide support for the assumption of an eight-stage theory of development. The FLCM serves several useful functions. These include: (a) substantially improving understanding of the eight developmental life stages proposed by Erikson, and (b) the use of it as a tool for timing of interventions. The next logical step would be to begin employing FLCM with treatment programs to enable clinicians to more effectively utilize processes of change.

\subsection{Erikson's Eight Developmental Life Stages}

The present research provides an important biopsychological basis for Erikson's eight-stage theory of development. The FLCM improves on existing classification efforts (Rose, 1991; Waskom, 1972) by linking the Fibonacci sequence to the 24-hour day/night cycle, which all organisms have adapted their behavior, physiology, and metabolism. Erikson's perspective on development can be criticized for lacking sufficient complexity to represent the epigenetic mechanisms involved. The FLCM provides a more complex conceptualization of development in which emphasis is placed on the interaction between the Fibonacci sequence, shifts in biological 
energy, and a series of psychosocial crises.

Despite the fact that Erikson's eight stages of psychosocial development have been highly influential in the understanding of human development, Erikson's theory has been criticized for taking as axiomatic a Western cultural context (Kahn, Zimmerman, Csikszentmihalyi, \& Getzels, 1985). It is argued the tasks or dilemmas to be solved at each stage of life are oriented to Western society and non-Western cultures may demonstrate different developmental trajectories. Focusing on life tasks with evolutionary significance links into the work of evolutionary psychology (Buss, 1995; Cosmides, Tooby \& Barkow, 1992) and has the advantage of resulting in a definition that is universal rather than culturally bound. Life tasks that were imposed by our ancestral environment are more universal - problems that affect all humans because they derive from a common human nature. Life tasks that have evolutionary significance are relevant to a stage theory based on the Fibonacci sequence because it offers the possibility of developing a theory that is universally applicable.

\subsection{Phase Transitions and Interventions}

Dynamic systems theory has received increased interest in the past few decades in views of development (van Geert, 2011, 2012). The fundamental premise is that dynamic systems theory considers attributes of all dynamic systems. Thus, assuming the human person is also a dynamic system, dynamic systems concepts can also explain human behavioral patterns. Therefore, dynamic systems theory offers a theoretical model with which to understand human development. The dynamic systems view of development "considers the origins and functions of variability as absolutely central for understanding change" (Thelen \& Smith, 1994, p. 67).

Dynamic systems transform through structural changes - a reorganization of attractor states referred to as a phase transition. During a phase transition new attractors emerge to produce new stable behavioral patterns. This transformation requires the prior stable configuration to break down. This transition period, consequently, reflects a brief rise in the unpredictability of the system as behavior becomes unstable and more variable. Numerous developmental transitions display attributes of a phase transition, such as changes in walking behavior of infants (Thelen \& Ulrich, 1991), socioemotional development (Lewis, Zimmerman, Hollenstein, \& Lamey, 2004), language (Bassano \& van Geert, 2007), and parent-adolescent relations (Granic, Hollenstein, Dishion, \& Patterson, 2003).

Phase transitions make a system more responsive to perturbations because of the temporary instability. Therefore, during these periods external factors have the greatest impact. This feature has two significant consequences for development. First, developmental phase transitions are often vulnerable periods. Second, developmental phase transitions could represent ideal times for treatment because the system (individual) is already in change. Thus, the most effective time for treatment interventions might be during a developmental phase transition (Granic, 2005). These two implications indicate that determining the occurrence of phase transitions in human development is essential since they permit researchers and clinicians to more effectively utilize change processes.

Normative stage transitions may represent a time during which, because of biopsychological processes, the organization among system parts breaks down, prior attractors become unstable, and new patterns of behavior have the possibility of arising (Granic, 2005). Research shows the quality of a person's psychological health can be contingent on the point in the life cycle. For example, in a cross-national study a significant U-shaped effect was found for age such that happiness levels appeared to diminish from young adulthood to middle age, reaching a minimum around age 48 1/2, and then increasing during older adulthood (Blanchflower \& Oswald, 2008). Depression has been described as an attractor, a set toward which a dynamical system evolves over time (Johnson \& Nowak, 2002). Thus, depression can be viewed as an indicator of a phase transition since a shift from one attractor to another defines a phase transition.

Fibonacci numbers can be used for simulation of self-organizing systems (Stakhov, 2009). Significantly, the demonstration that the Fibonacci sequence appears within the Feigenbaum scaling of the period doubling cascade to chaos suggests a correlation between the Fibonacci sequence and the onset of chaos and turbulence in nonlinear systems (Linage, Montoyaa, Sarmientob, Showalter, \& Parmananda, 2006). The FLCM could explain why well-being bottoms out at age $48 \mathrm{1} / 2$ around the world (Blanchflower \& Oswald, 2008). In terms of biopsychological development, the FLCM predicts age 48 as a phase transition between middle adulthood (29-48) and older adulthood (48-78+). Psychosocial stress and depressive symptoms may be aspects of dynamical instability representing the shift from one developmental stage (or attractor) to the next. The FLCM can also be used to calculate secondary transitions within the eight primary transition periods. This has immense potential in its application for designing treatment interventions that aim to more effectively utilize processes of change. 


\subsection{Summary}

The Fibonacci Life-Chart Method (FLCM) provides a biopsychological basis for Erikson's life-cycle theory and eight stages founded on the occurrence of Fibonacci numbers in biological cell division and self-organizing systems. This paper can contribute to research on identifying the origins of disequilibrium in human development that is central for understanding change. Within a dynamic systems theory framework, the onset of disequilibrium is a signal that change is happening, which may allow prevention or improvement of psychological symptoms through early intervention.

It should be pointed out the FLCM is preliminary and necessarily incomplete. It is acknowledged Fibonacci numbers may be represented by alternative numeric designations (minutes, seconds, hours, years) in future time projections. However, it is hoped the FLCM has identified the 24-hour day as a unique factor and, critically, the biological and mathematical relations of the day/night cycle suggest how additional mechanisms could be integrated within this method.

One cannot conclude from this study that the FLCM is predictive. The definitive validation would be a longitudinal study with long-term follow-up. This would allow correlations to be made between the FLCM and functional outcome. Future projects could include: (a) using the FLCM to identify populations requiring early intervention, and (b) conducting a longitudinal study to establish the power of the FLCM to improve the efficacy of clinical treatment programs.

\section{Conclusion}

This study shows a new method, called the Fibonacci Life-Chart Method (FLCM), produces a developmental pattern characterized by eight recognizable stages. The available empirical and conceptual evidence is consistent with an eight-stage theory of development. It is hoped this research will contribute to a better understanding of Erikson's eight developmental stages and the dynamic systems view of development. Dynamic systems theory considers disorder, unpredictability, and lack of control as normal parts of phase transitions. Understanding and determining the occurrence of phase transitions in human development can lead not only to a better understanding of the etiology of psychological disorders associated with psychosocial stress, but also to a potential avenue for early intervention and perhaps, ultimately, prevention.

\section{References}

Bassano, D., \& van Geert, P. (2007). Modeling continuity and discontinuity in utterance length: A quantitative approach to changes, transitions and intra-individual variability in early grammatical development. Developmental Science, 10, 588-612. http://dx.doi.org/10.1111/j.1467-7687.2007.00629.x

Blanchflower, D. G., \& Oswald, A. J. (2008). Is well-being U-shaped over the life cycle? Social Science \& Medicine, 66, 1733-1749. http://dx.doi.org/10.1016/j.socscimed.2008.01.030

Buss, D. M. (1995). Evolutionary psychology: A new paradigm for psychological science. Psychological Inquiry, 6(1), 1-30. http://dx.doi.org/10.1207/s15327965pli0601_1

Cosmides, L., Tooby, J., \& Barkow, L. (Eds.). (1992). The adapted mind: Evolutionary psychology and the generation of culture. New York, NY: Oxford University Press.

Erikson, E. H. (1982). The life cycle completed. New York: Norton.

Goldenberger, A. L., West, B. J., Dresselhaus, T., \& Bhargava, V. (1985). Bronchial asymmetry and Fibonacci scaling. Cellular and Molecular Life Sciences, 41, 1537-1538. http://dx.doi.org/10.1007/BF01964794

Granic, I. (2005). Timing is everything: Developmental psychopathology from a dynamic systems perspective. Developmental Review, 25, 386-407. http://dx.doi.org/10.1016/j.dr.2005.10.005

Granic, I., Hollenstein, T., Dishion, T. J., \& Patterson, G. R. (2003). Longitudinal analysis of flexibility and reorganization in early adolescence: A dynamic systems study of family interactions. Developmental Psychology, 39, 606-617. http://dx.doi.org/10.1037/0012-1649.39.3.606

Hoggatt, V. E., \& Lind, D. A. (1969). The dying rabbit problem. The Fibonacci Quarterly, 7(5), 482-487.

Huntley, H. E. (1969). Fibonacci and the atom. Fibonacci Quarterly, 7(5), 523-524.

Johnson, S., \& Nowak, A. (2002). Dynamical patterns in bipolar depression. Personality and Social Psychology Review, 6, 370-379. http://dx.doi.org/10.1207/S15327957PSPR0604_12

Jovanovic, R. (2003). Cell division and the Pascal triangle. Retrieved from $\mathrm{http}: / /$ milan.milanovic.org/math/english/division/division.html 
Kahn, S., Zimmerman, G., Csikszentmihalyi, M., \& Getzels, J. W. (1985). Relations between identity in young adulthood and intimacy at midlife. Journal of Personality and Social Psychology, 49, 1316-1322. http://dx.doi.org/10.1037/0022-3514.49.5.1316

Lewis, M. D., Zimmerman, S., Hollenstein, T., \& Lamey, A. V. (2004). Reorganization in coping behavior at 1 1/2 years: Dynamic systems and normative change. Developmental Science, 7, 56-73. http://dx.doi.org/10.1111/j.1467-7687.2004.00323.x

Linage, G., Montoya, F., Sarmiento, A., Showalter, K., \& Parmananda, P. (2006). Fibonacci order in the period-doubling cascade to chaos. Physics Letters, A359, 638-639. http://dx.doi.org/10.1016/j.physleta.2006.07.036

Meisner, G. (May, 2012). Repeating pattern in the Fibonacci series. Retrieved from http://www.goldennumber.net/fibonacci-24-pattern/

Prokopchuk, V. S. (1983). Mathematical model of the morphogenesis of tumor nodules. Arkhiv Patologii, 45, 55-61.

Roenneberg, T., \& Foster, R. G. (1997). Twilight times: Light and the circadian system. Photochemistry and Photobiology, 66, 549-561. http://dx.doi.org/10.1111/j.1751-1097.1997.tb03188.x

Rose, N. (1991). Design and development of wholeness: Waskom's paradigm. The Educational Forum, 55, 243-259. http://dx.doi.org/10.1080/00131729109335650

Spears, C. P., \& Bicknell-Johnson, M. (1998). Asymmetric cell division: Binomial identities for age analysis of mortal vs. immortal trees. Applications of Fibonacci Numbers, 7, 377-391.

Stakhov, A. P. (2009). The mathematics of harmony: From Euclid to contemporary mathematics and computer science. Singapore: World Scientific Publishing.

Thelen, E., \& Smith, L. B. (1994). A dynamic systems approach to the development of cognition and action. Cambridge, MA: MIT Press.

Thelen, E., \& Ulrich, B. D. (1991). Hidden skills: A dynamic systems analysis of treadmill stepping during the first year. Monographs of the Society for Research in Child Development, 56(1, Serial No. 223). http://dx.doi.org/10.2307/1166099

van Geert, P. (2011). The contribution of complex dynamic systems to development. Child Development Perspectives, 5(4), 273-278. http://dx.doi.org/10.1111/j.1750-8606.2011.00197.x

van Geert, P. (2012). Dynamic systems. In B. Laursen, T. D. Little, \& N. A. Card (Eds.), Handbook of developmental research methods (pp. 725-741). New York: Guilford.

Wahl, M. (1988). A mathematical mystery tour. Tucson, AZ: Zephyr Press.

Waskom, J. (1972). The magic of design and other unpublished talks. Loveland, Colorado. 